\title{
Evaluation and impact of different biomarkers for early detection of hepatocellular carcinoma
}

\author{
Jan Best ${ }^{1}$, Svenja Sydor ${ }^{2}$, Lars P. Bechmann², Ali Canbay ${ }^{2}$ \\ 'Department of Gastroenterology, Hepatology and Infectious Diseases, Otto-von-Guericke University, University Hospital \\ Magdeburg, Magdeburg 39120, Germany. \\ ${ }^{2}$ Department of Internal Medicine, University Hospital Knappschaftskrankenhaus, Ruhr-University Bochum, Bochum 44892, \\ Germany.
}

Correspondence to: Dr. Jan Best, Department of Gastroenterology, Hepatology and Infectious Diseases, Otto-von-Guericke University, University Hospital Magdeburg, Leipziger Str. 44, Magdeburg 39120, Germany. E-mail: jan.best@med.ovgu.de

How to cite this article: Best J, Sydor S, Bechmann LP, Canbay A. Evaluation and impact of different biomarkers for early detection of hepatocellular carcinoma. Hepatoma Res 2020;6:62. http://dx.doi.org/10.20517/2394-5079.2020.56

Received: 2 Jun 2020 First Decision: 24 Jun 2020 Revised: 7 Jul 2020 Accepted: 9 Jul 2020 Published: 1 Sep 2020

Academic Editor: Guido Guenther Gerken Copy Editor: Cai-Hong Wang Production Editor: Jing Yu

\begin{abstract}
Worldwide, hepatocellular carcinoma (HCC) is a frequent complication of liver diseases and remains a major cause of cancer-related mortality. In addition, the prevalence of nonalcoholic steatohepatitis (NASH) as prerequisite of hepatocarcinogenesis, even in the absence of cirrhosis, is rising rapidly. The early detection of HCC has been crucial in improving the survival outcomes of those patients. However, in the mostly obese NASH population, diagnostic sensitivity of ultrasound-based HCC screening approaches is limited. On the other hand, biomarkers for HCC show promising potential to improve early detection, providing reproducible, investigator-independent results that can be used either alone or integrated with other biomarkers for scoring models. In the past, validation has been limited due to a lack of prospective longitudinal cohort studies. At present, large-scale retrospective phaseIII- biomarker- development gives hope for the availability of biomarker-based screening approaches in the near future. This review focuses on the potential impact of biomarkers on surveillance strategies, potentially allowing for earlier HCC diagnosis.
\end{abstract}

Keywords: Nonalcoholic steatohepatitis, hepatocellular carcinoma, alpha fetoprotein, AFP-L3, des-gammacarboxy-prothrombin, Gender, Age, GALAD-score, Glypican-3, microbiome 


\section{INTRODUCTION}

Globally, hepatocellular carcinoma (HCC) is the sixth most frequent malignancy and the second most common cause of cancer-related death ${ }^{[1]}$. The global incidence of HCC has tripled since the $1980 \mathrm{~s}^{[2]}$. Despite the availability of numerous locoregional therapies and a plethora of novel systemic treatment options, the overall survival of HCC remains relatively poor ${ }^{[3]}$. Furthermore, incidence of HCC in the western world is rising with hepatic steatosis being seen as the major risk factor [Figure 1]. Nonalcoholic fatty liver (NAFL; i.e., hepatic steatosis without significant inflammation) and nonalcoholic steatohepatitis (NASH; i.e., hepatic steatosis associated with hepatic inflammation and hepatocellular ballooning) in particular have increasingly been recognized as risk factors for HCC. Previously, these cases have frequently been classified as cryptogenic cirrhosis ${ }^{[5]}$. Moreover, a significant proportion of patients develops HCC in the absence of liver cirrhosis predominantly in the case of predisposing chronic hepatitis B or NASH ${ }^{[6]}$. Nevertheless, multiple HCC surveillance guidelines do not sufficiently recognize NASH as major risk factor of hepatocarcinogenesis. Additionally, ultrasound for detection of smaller lesions, such as in early stage HCC, lacks sensitivity and is further impaired when there is underlying cirrhosis, steatosis, or obesity. To address those aforementioned insufficiencies, the definition of patients at risk has to be more concisely defined and further prospective trials have to elucidate whether ultrasound alone has potential to detect a sufficient proportion of HCC at stages when curative treatment options are still available. Several recent trials clearly indicate that biomarker-based surveillance algorithms have potential to complement or even surpass ultrasound as a surveillance strategy. This review aims to provide an overview of current biomarkers with utility in HCC detection and how they could be implemented into current HCC early detection programs.

\section{EARLY HCC DIAGNOSIS DETERMINES PATIENT PROGNOSIS}

In patients participating in HCC early detection programs, an initial diagnosis is made in less advanced stages, which results in a clear survival benefit. Successful surveillance, however, requires a reliable screening method and a definition of the risk population based on medical needs.

Targeted HCC monitoring anticipates offering curative intended therapeutic procedures, such as liver resection or transplantation, to the highest possible proportion of patients upon their initial HCC diagnosis. Unfortunately, HCC is diagnosed in these early stages only in a minority of patients ${ }^{[7]}$. In a representative German singlecenter cohort, between 1998 and 2009, only 23.5\% of over a thousand HCC patients were in an early stage of HCC [Barcelona Clinic Liver Cancer (BCLC) 0/A] at initial diagnosis. Accordingly, less than half of the patients received curative therapies and the median overall survival was only 16 months ${ }^{[8]}$.

The above-mentioned data are consistent with large international trials where only $10 \%-23 \%$ of the patients were curatively treatable at first diagnosis ${ }^{[9-12]}$. Consequently, only $10 \%-39 \%$ of those patients survived at least one year after diagnosis ${ }^{[9-14]}$.

Since HCC is better suited for early detection programs than most other cancers, and since the risk population to be screened could be well defined, the currently available data is particularly discouraging. International guidelines recommend regular examinations of symptom-free risk patients according to certain criteria.

\section{INTERNATIONAL GUIDELINES PROVIDE DIFFERENT HCC SURVEILLANCE RECOMMENDATIONS}

Globally, there are marked regional variations in the algorithms for HCC early detection [Table 1]. The guideline of the German Society for Digestive and Metabolic Diseases (DGVS) recommends that patients with liver cirrhosis of any etiology, as well as patients with chronic hepatitis B or NASH in the absence of cirrhosis, undergo liver ultrasound. Optionally, the liver tumor marker $\alpha$-fetoprotein (AFP) can be 


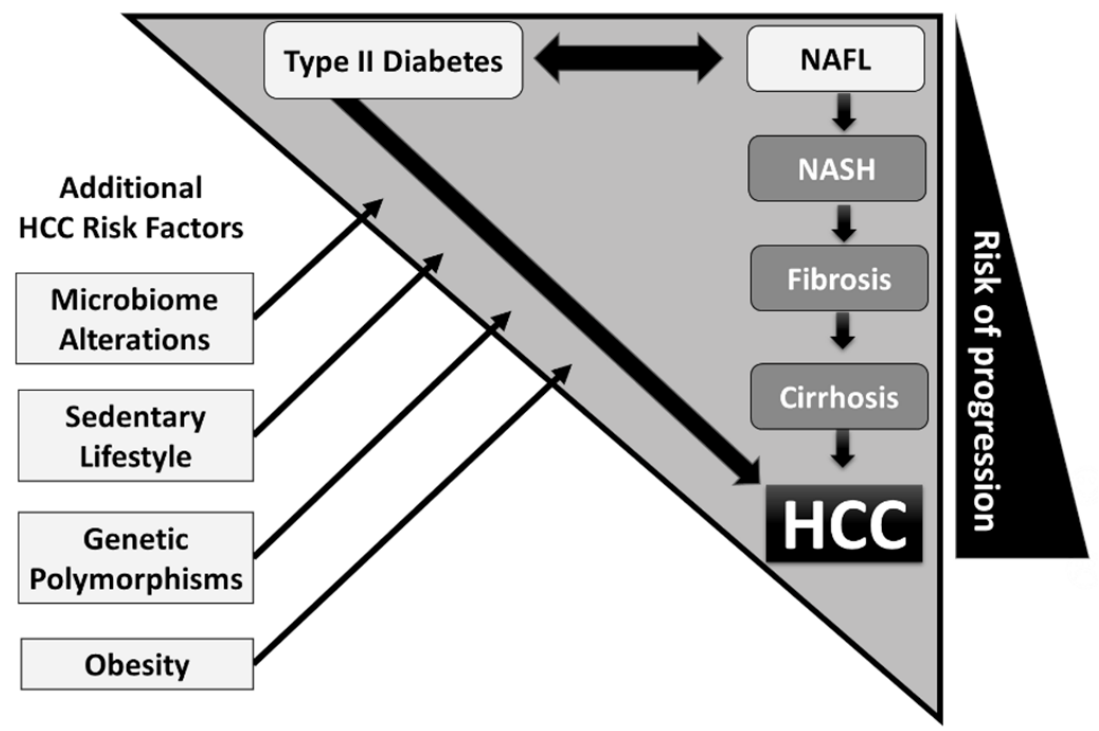

Figure 1. Triangle of hepatocarcinogenesis in metabolic syndrome: hepatocellular carcinoma (HCC) risk in nonalcoholic steatohepatitis (NASH) significantly increases with progression of liver fibrosis. Independently, type II diabetes mellitus promotes progression from nonalcoholic fatty liver (NAFL) to NASH, but needs to be recognized as an individual predisposing HCC-risk factor since peripheral insulin resistance may promote hepatocarcinogenesis even in the absence of cirrhosis ${ }^{[4]}$. Microbiome alterations, sedentary lifestyle, genetic polymorphisms, and obesity represent additional factors aggravating HCC risk in the NASH population

Table 1. Overview of international HCC surveillance recommendations

\begin{tabular}{lll}
\hline Society & \multicolumn{1}{c}{ Risk group } & \multicolumn{1}{c}{ Procedure } \\
\hline DGVS $^{[15]}$ & Liver cirrhosis of all etiologies: chronic HBV and NASH & US with or without AFP every 6 months, \\
AASLD ${ }^{[16]}$ & $\begin{array}{l}\text { Liver cirrhosis of all etiologies; chronic HBV depending on ethnical } \\
\text { background, age, and genetic background } \\
\text { Liver cirrhosis of all etiologies at CTP stage A and B or CTP stage C } \\
\text { EASL/EORCT }{ }^{[17]}\end{array}$ & US with or without AFP every 6 months \\
& $\begin{array}{l}\text { if listed for oLT; chronic hepatitis B or active hepatitis; chronic HCV } \\
\text { with advanced (F3) fibrosis }\end{array}$ & \\
APASL $^{[18,19]}$ & $\begin{array}{l}\text { Liver cirrhosis, chronic HBV and/or HCV } \\
\text { JSH }^{[20]}\end{array}$ & High risk: Liver cirrhosis of all etiologies; chronic HBV and/or HCV \\
& Very high risk: Liver cirrhosis with chronic HBV and/or HCV & US and AFP every 6 months \\
& & US, AFP, AFP-L3 and DCP every 6 months DCP every 3-4 months \\
\hline
\end{tabular}

DGVS: German Society of Gastroenterology and Metabolic Diseases; EASL: European Association for the Study of the Liver; AASLD: American Association for the Study of Liver Disease; APASL: Asian Pacific Association for the Study of the Liver; JSH: Japanese Society of Hepatology; HBV: hepatitis B virus; NASH: non-alcoholic Steatohepatits; HCV: hepatitis C virus; US: ultrasound; AFP: alpha fetoprotein; AFP-L3: Lektin reactive $\alpha$-Fetoprotein; DCP: Des-gamma-carboxy prothrombin also known as Protein-Induced-by-VitaminK-Absence-or-Antagonist-II (PIVKA II); CTP: Child Turcotte Pugh

determined. The sonographic examinations should be carried out according to quality criteria of the German Society for Ultrasound in Medicine (DEGUM) ${ }^{[15]}$.

While the guidelines of the the American Society for the Study of the Liver (AASLD) and the European Association for the Study of the Liver (EASL) do not recommend an AFP determination at all, or only recommend it on an optional basis, the Asian Pacific Association for the Study of the Liver (APASL) proposed a combination of regular ultrasound (US) and AFP determination. All the aforementioned guidelines recommend those examinations every 6 months. The Japanese Society for Hepatology (JSH) also recommends US in combination with complimentary determination of three different biomarkers, namely AFP, lectin-reactive- $\alpha$-fetoprotein (AFP-L3), and des-gamma-carboxy-prothrombin (DCP). In contrast to all other guidelines, JSH discriminates between high risk and very high risk groups and therefore recommends screening every 6 months and every 3 months, respectively, as depicted in Table 1. 
The limitations of ultrasound scanning have been explicitly recognized in previous AASLD guidelines which state "performance characteristics have not been well defined in cirrhotic livers" and "some patients, particularly the obese, are not good candidates (for surveillance) despite their risk" ${ }^{[21-25]}$. To address this insufficiency, a recent large meta-analysis, which included thirty-two studies comprising 13,367 patients, characterized sensitivity of imaging with or without AFP for detection of HCC in cirrhotic patients. The authors concluded that US alone has a low sensitivity to detect early stage HCC in patients with cirrhosis and thus the addition of AFP to US significantly increased the sensitivity of early HCC detection ${ }^{[26]}$. The latest AASLD guidelines emphasize the importance of determining whether other serum biomarkers (specifically AFP-L3 and DCP) might complement AFP and US in the surveillance setting ${ }^{[16]}$.

\section{NASH AS MAIN RISK FACTOR OF INCREASING HCC INCIDENCE}

The global prevalence of nonalcoholic fatty liver disease (NAFLD) is estimated at $25 \%{ }^{[27]}$. In up to one third of all NAFL patients, progression to NASH can occur, which in turn may lead to higher-grade fibrosis on the basis of inflammation and, in the final stage, to cirrhosis and $\mathrm{HCC}^{[28,29]}$. A recent metaanalysis conducted by Younossi et al. ${ }^{[13]}$ even reported a global prevalence of NASH among biopsied NAFLD patients that was almost double the previous studies' findings at $59.1 \%$. According to the latest epidemiological studies, NASH is regarded as the main cause of the steadily increasing incidence of HCC in western industrialized nations ${ }^{[30,31]}$. An analysis of the American liver transplant registry from 2002 to 2007 showed that the prevalence of HCC patients waiting for a liver transplant increased by $15.6 \%$ in the past 15 years, from $6.4 \%$ in 2002 to $22 \%$ in 2017 . NASH became the fastest growing cause of HCC. The proportion of NASH as the cause of HCC increased 8.5-fold during the study period, from $2.1 \%$ in 2002 to $17.9 \%$ in $2017^{[31]}$. It should be noted here that many cases of HCC arise due to predisposing NASH in the absence of cirrhosis, a population that has so far not been internationally screened for the purpose of early detection of HCC. About $20 \%$ of all NASH-associated HCC cases occurred in the absence of cirrhosis ${ }^{[32]}$.

A German single-center study from 2015, which included 1,119 HCC patients of all etiologies, showed that there are relevant differences between patients with HCC with underlying NASH compared to those with other hepatopathies. Patients with NASH-related HCC were older than those with other predisposing hepatopathies (67.6 years $v s .65$ years) when they were first diagnosed. In addition, the NASH-HCC cohort showed a higher prevalence of obesity $(31.1 \%$ vs. $14.7 \%)$ and diabetes mellitus type II (T2DM) (66.7\% vs. $37.85 \%$ ) [Figure 1]. Interestingly, NASH-associated HCC shows a trend towards a higher frequency of multifocality ( $80 \% v s .69 .7 \%)$ with overall larger lesions $(6 \mathrm{~cm} v s .4 .8 \mathrm{~cm})$ and a tendency towards an increased rate of extrahepatic metastasis at time of initial diagnosis. It is also important to note that the NASH-HCC patients had better global liver function despite a higher tumor burden when diagnosed for the first time. It can be postulated that later HCC diagnosis may be the result of less intensive screening efforts due to lesser extent of hepatic deterioration in NASH patients compared to other liver diseases ${ }^{[33]}$.

\section{DO BIOMARKERS ENHANCE HCC EARLY DETECTION?}

For clinical routine, the diagnostic significance of ultrasound of the liver as part of the HCC screening may exhibit distinct limitations. This includes the comparability with preliminary examinations due to changing investigators and ultrasound devices. Predominantly in the cirrhotic or steatotic liver, small lesions are detected in a limited extent. Determination of HCC biomarkers such as AFP have the advantage of being independent of the investigator. Laboratory tests are subject to strict quality guidelines and deliver reproducible results. For this reason, the addition of AFP is recommended in some guidelines to supplement the US. In the high-risk cohort suffering from chronic viral hepatitis B or C relevant for HCC screening, however, the hepatic inflammation may lead to a false positive AFP value elevation which significantly attenuates the specificity, especially in this scenario ${ }^{[34,35]}$. In contrast, if the AFP cut-off values are set higher to improve specificity, $40 \%$ of the early stages of HCC are not detected by AFP ${ }^{[36]}$. 
In order to achieve a sensitivity superior to the combination of US and AFP while preserving high specificity in early HCC detection, a large number of studies examined the suitability of different biomarkers, such as Glypican-3, AFP-L3, and DCP for HCC surveillance ${ }^{[37-40]}$. The focus during recent years has been on validation of AFP, AFP-L3, and DCP which are already available as certified laboratory tests.

\section{AVAILABLE HCC-BIOMARKERS AND DIAGNOSTIC MODELS AFP and AFP-L3}

The overall AFP, which is usually determined in the clinical routine, consists of the three different isoforms: AFP-L1, AFP-L2, and AFP-L3. AFP is a fetal glycoprotein that can be produced later in life when the hepatocytes are in the process of malignant transformation ${ }^{[41]}$. According to various studies, the sensitivity of AFP in HCC detection is between $39 \%$ and $65 \%$ and the specificity varies between $76 \%$ to $94 \%{ }^{[42]}$.

AFP-L3 is a AFP variant that binds to the lectin molecule "Lens culinaris agglutinin" and, in contrast to the overall AFP, is HCC-specific. While the AFP-L3 fraction is produced exclusively by malignant transformed hepatocytes, an AFP-L1 elevation, the non-glycosylated AFP main fraction, can also be caused by viral hepatitis and in this scenario is responsible for an incorrectly increased total AFP level ${ }^{[43,44]}$. With a cut-off of $15 \%$, sensitivities between $75 \%-96.6 \%$ and specificities of $90 \%-92 \%$ have been described for AFP-L $3^{[45,46]}$.

\section{Des-gamma-carboxy prothrombin}

Des-gamma-carboxy prothrombin (DCP), also known as Protein-Induced-by-Vitamin-K-Absenceor-Antagonist-II (PIVKA II), is a precursor of prothrombin and is formed in the context of hepatocarcinogenesis due to an impaired vitamin K metabolism. Here, the carboxylation of prothrombin is so impaired that the serum concentration of the DCP increases. Sensitivities between $48 \%$ and $62 \%$ and specificities between $81 \%$ and $98 \%$ have been described for the DCP, making DCP a more specific marker than AFP, albeit with lower sensitivity ${ }^{[47-49]}$.

\section{Combination of AFP, AFP-L3 and DCP}

Various clinical trials have clearly demonstrated that there were no correlations among the results of AFP, AFP-L3, and DCP. Thus, some HCC cases can be positive for only one marker at a time while negative for the others. In clinical practice, this means that the combination of the above biomarkers leads to a gradual increase in sensitivity.

Especially when AFP remains the only available marker in clinical routine, the complementary use of AFP-L3 and DCP represents an additional diagnostic option. In a retrospective Japanese single-center study with 270 AFP-negative HCC patients, it was demonstrated that the majority of patients with positive AFP-L3 and/or DCP findings were correctly recorded ${ }^{[50]}$.

\section{GALAD model}

The aforementioned triple combination of the biomarkers AFP, AFP-L3, and DCP demonstrated superior detection of HCC compared to their individual utilization with no significant decrease in specificity in an Asian patient cohort ${ }^{[51,52]}$. For further optimization, a statistically based model called GALAD score was developed a few years ago and was extensively validated in several international studies. It is a diagnostic algorithm based on rigorous statistical analysis. The formula is calculated on the measured absolute values of the three markers instead of defining cut-off levels. Thus, they are considered as continuous variables rather than categorical. Gender and age information are also included since older age and male sex represent independent HCC risk factors ${ }^{[53,54]}$. A GALAD point value of -0.63 serves as a cut-off value for optimal sensitivity and specificity regardless of the BCLC stage. Using this model in a British cohort, the GALAD model achieved an overall AUROC (area under the receiver operating characteristic curve) of 0.97 in detection of all BCLC stages, and early stage HCC (BCLC 0/A) was detected with an AUROC of 
0.92 and a corresponding sensitivity of $86 \%$ and specificity of $89 \%{ }^{[55]}$. A subsequent international multicenter validation study comprising 6,834 patients of different etiologies of HCC achieved an AUROC for GALAD of consistently $>0.90$, confirming the efficacy of this model ${ }^{[56]}$. A following German singlecenter study observed that the GALAD score, even in BCLC 0/A stage HCC, achieved an AUROC of 0.92, again demonstrating superiority in HCC detection compared to the triple biomarker combination of AFP, AFP-L3 and DCP without considering gender and age ${ }^{[57]}$.

To further address the inadequate performance of US-based HCC early detection in NASH, where obesity and sound artifacts due to steatosis further attenuate the diagnostic performance, the utility of the GALAD score in the detection of NASH-associated early HCCs had been tested specifically in this this high-risk collective. In a retrospective German multi-center cohort (8 centers) study, the GALAD score was able to detect NASH-HCC patients with an AUROC of 0.96, significantly better than the performance of the biomarkers alone [AFP (AUROC 0.88), AFP-L3 (AUROC 0.86), or DCP (AUROC, 0.87)]. Even for NASH patients in early HCC stages (within the Milan criteria), the GALAD score achieved an AUROV of 0.91. Furthermore, in a prospective Japanese cohort study, it was demonstrated that the average GALAD score in those patients who developed HCC during the observation period was already significantly elevated up to 1.5 years before the initial diagnosis of HCC. The GALAD scores of these HCC patients rose above the cutoff value of 0.63 approximately 200 days before first diagnosis ${ }^{[58]}$.

This implies that the GALAD model is quite suitable for early detection of HCC of all etiologies, even in NASH. However, a phase IV multi-center prospective study has yet to test whether the GALAD Score can be used in the future as an integral part of HCC screening algorithms in patients at risk.

\section{Osteopontin}

Osteopontin (OPN) is an integrin-binding phosphoprotein that is overexpressed in a variety of cancers including lung, breast, colon cancer, and $\mathrm{HCC}^{[39,59]}$. At a low level, it is also secreted by biliary epithelial cells. OPN mediates cell signaling that controls inflammation as it is the case in hepatitis, HCC tumor progression, and metastasis ${ }^{[60]}$. Hepatocarcinogenesis results in elevated OPN levels compared to those patients with chronic liver disease in the absence of $\mathrm{HCC}^{[61]}$. In a meta-analysis investigating the efficacy of OPN in HCC detection, the sensitivity and specificity of elevated OPN levels have been reported between $75 \%-87 \%$ and $62 \%-82 \%$, respectively ${ }^{[62]}$. In a phase III validation study, OPN outperformed AFP for HCC detection with an AUROC of 0.73 [95\%CI: 0.62-0.85] vs. AUROC of 0.68 [95\%CI: 0.54-0.82], respectively. The combined utilization of AFP and OPN resulted in a sensitivity of $82 \%$ and specificity of $77 \%$ for HCC detection; however, the number of patients at BCLC stage 0/A was limited in this study ${ }^{[63]}$.

\section{Glypican-3}

Glypican-3 (GPC-3), is a heparin sulfate proteoglycan playing a pivotal role in cell proliferation and tumor suppression, representing a potential biomarker for the diagnosis of HCC. GPC-3 binds to growth factor receptors and is involved in cell proliferation and tumor suppression ${ }^{[64]}$. In healthy hepatocytes it is absent, but during hepatocarcinogenesis it is upregulated, and it is assumed to participate in the canonical Wnt- signaling growth pathway ${ }^{[65,66]}$. GPC-3 is present in approximately $33 \%$ of HCC patients that were seronegative for both DCP and $\mathrm{AFP}^{[67]}$. A meta-analysis found that GCP-3 had a sensitivity of $55.1 \%$ and a specificity of $97 \%{ }^{[68]}$. AFP and GCP-3 in concert achieved a sensitivity of $76 \%$ even at early stage HCC. In light of these findings, GPC-3 has been proposed to be a complementary serologic biomarker to AFP due to the ability of GPC-3 to accurately distinguish between patients with small, well differentiated HCC and those with underlying cirrhosis ${ }^{[69]}$. 


\section{HCC SURVEILLANCE BASED ON COMBINATION OF BIOMARKERS AND ULTRASOUND Combination of AFP and ultrasound}

The latest HCC guidelines have recently shown a tendency to omit biomarker-based diagnostics in favor of sole ultrasound examinations. In a large American meta-analysis of US, the sensitivity and specificity of US in HCC detection was analyzed with and without additional AFP determination in an HCC highrisk patient group (32 trials/13,367 patients). Ultrasound alone detected an HCC across all stages with a sensitivity of $84 \%$ when carried out in accordance with regional guidelines, but there was a dramatic drop in sensitivity to $47 \%$ in early stage HCC. The combination of US with AFP was able to improve the sensitivity in the early detection of HCC to at least $63 \%{ }^{[21]}$. This clearly indicates that ultrasound alone has low sensitivity in detecting early stage HCC in patients with cirrhosis. Hence, the addition of AFP to ultrasound may significantly increase the sensitivity of early HCC detection in future.

\section{Combination of GALAD and ultrasound}

Recently, the GALAD model has also been validated in an American US cohort study [single-center cohort of $111 \mathrm{HCC}$ and 180 controls and a multi-center cohort of 233 early HCC and 412 cirrhosis patients from the Early Detection Research Network (EDRN) Phase 2 HCC Study] and the performance has been shown to be clearly superior to sonography for HCC detection. Here the AUROC of GALAD for HCC detection was 0.95 , which was clearly superior to the AUROC of ultrasound (0.82). The combination of GALAD and ultrasound (GALADUS score) achieved an AUC of 0.98, clearly superior to US or GALAD used solely ${ }^{[70]}$. These very promising data indicate that a combination of ultrasound and biomarker-based scores can significantly improve the performance of current surveillance strategies.

\section{MECHANISMS OF NASH-RELATED HEPATOCARCINOGENESIS AS POTENTIAL TARGETS FOR SURVEILLANCE}

Understanding the sequence from NAFLD to HCC and the impact of additive risks such as type II diabetes mellitus [Figure 1], genetic polymorphisms, and stool microbiome are increasingly becoming the focus of current research.

Inflammation per se, which defines NASH, is a clinically relevant trigger of carcinogenesis, even without the basis of cirrhosis of the liver. While an altered lipid and glucose metabolism contributes to hepatic steatosis in the context of the metabolic syndrome, the interplay of genetic variations, mitochondrial dysfunction, altered immune response, and an imbalance of the microbiome cause a progression of simple fatty liver to NASH, and in the "worst case" scenario, HCC.

\section{GENETIC FACTORS}

For certain gene polymorphisms, there is a direct relationship between the prevalence of NAFLD and the risk of progression to advanced NASH fibrosis.

The polymorphism of the patatin-like-phospholipase-domain-containing-3 (PNPLA3) gene leads to increased hepatic lipid accumulation and an alteration in retinol storage in the liver. Independent of potential disruptive factors such as body mass index (BMI), diabetes, and advanced fibrosis, there is a 3 -fold increased HCC risk due to PNPLA ${ }^{[71]}$.

The polymorphism of the transmembrane 6-superfamily member 2 (TM6SF2) gene manifests itself as a transport disorder of pre-VLDL (very low-density lipoprotein) particles. There is a correlation with the extent of steatosis and progression of fibrosis in NASH, regardless of obesity, diabetes, and PNPLA3 genotype. The direct role of this TM6SF2 variant in hepatocarcinogenesis has not yet been fully elucidated; it is possibly the profibrogenic effect that indirectly promotes progression to $\mathrm{HCC}^{[72]}$. 
Genetic variability is not completely explained by these common aforementioned variants and many of the phenotypic differences potentially result from gene-environment interactions. NAFLD development and progression are also modulated by epigenetic factors, in particular microRNAs (miRNAs). At the posttranscriptional level, they control many complementary target mRNAs. Their dysregulation have a high predictive value in NAFLD development and progression ${ }^{[73,74]}$. Epigenetic changes, which cause aberrant DNA methylation, as well as the expression of various micro-RNAs (e.g., miR-21, miR-29, miR-23, miR-155, miR-221, miR-222, miR-106, miR-93, and miR-519) are additive drivers of carcinogenesis. There is a direct influence on the most relevant tumor-associated signal cascades [transforming growth factor beta, wingless and INT-1 (Wnt)/ $\beta$-catenin, mitogenactivated protein kinase, and phosphatidylinositol 3-kinase/AKT/ mammalian target of rapamycin] $]^{[75]}$.

\section{GUT MICROBIOME ANALYSES AS FUTURE PREDICTORS OF HCC RISK}

Distinct changes or shifts in the composition of intestinal bacteria have been described for different intestinal metabolic and inflammatory diseases ${ }^{[76-78]}$. Shifts of certain bacterial strains may also affect the formation of different bacterial-derived metabolic active components such as short-chain fatty acids, bile acids, ethanol, cytokines, or other inflammatory metabolites that may affect the host and possibly promote cancer-related risk factors or diseases ${ }^{[79]}$. The microbiota of an obese host has an impact on bodyweight and gut permeability, and it affects the release of gut-derived components that may also influence the progression of inflammatory mechanisms and promote the formation of cancer. Mouse models of fecal microbiota transfer (FMT) from obese to lean animals could show that microbiota affect bodyweight ${ }^{[80]}$. FMT is a potential therapeutic tool that has been used to treat Clostridium difficile infections ${ }^{[81]}$. In mouse studies, FMT was shown to be a useful treatment to increase abundances of beneficial bacterial groups such as Christensenellaceae and Lactobacillus to alleviate the progression of NASH-development ${ }^{[82]}$.

The modulation of the gut microbiota by FMT to treat or study different metabolism-related diseases has been taken into account for many years ${ }^{[83]}$. It has been shown that antibiotic treatments, in order to modulate the gut microbiota, may reduce the risk of hepatic carcinogenesis ${ }^{[84]}$. In a high fat diet mouse model of NASH, antibiotic treatments were associated with a reduction of toxic secondary bile acids ${ }^{[85]}$.

NAFL and especially its progressive inflammatory form NASH are often related to the formation of HCC. Here, nutrition, metabolic disturbances, and related comorbidities such as diabetes may influence the composition of the gut microbiota. Changes in the abundances of different bacterial groups have been described within different patient groups.

The metabolism of certain bacterial groups affects the mucosal barrier, hepatic inflammation, fibrogenesis, and tumorigenesis ${ }^{[86]}$. The gut microbiota has an impact on energy balance, altering the uptake of calories derived from food and even alcohol ${ }^{[87]}$. Emerging data indicate that certain characteristic changes in the gut microbiome are associated with NAFLD and even with cirrhosis, which is a main driver of HCCdevelopment ${ }^{[76,88,89]}$. It has not only been shown in NAFLD-related HCC, but also in viral hepatitis-related HCC (hepatitis B) that makes a modification of specific gut microbiota, a potential therapeutic option for $\mathrm{HCC}^{[90]}$.

In a study comparing NASH and NASH-HCC patients with or without cirrhosis, alterations in bacterial groups regulating bile acid metabolism had an impact on hepatic fibrogenesis and liver injury. Alterations of the bile acid pool was accompanied with increased abundances of different bacterial strains, especially Lactobacilli and Bacteroides which were associated with changed liver stiffness and liver injury ${ }^{[1]]}$. In NASH patients, the abundance of bile salt hydrolase (an enzyme involved in deconjugation of bile acids) expressing bacteria is shifted, which leads to increased bile acid levels as well as an altered composition of the bile acid pool tending to an increased amount of secondary conjugated bile acids ${ }^{[92]}$. Changes within the 
bile acid composition can be related to advanced fibrosis in NASH-HCC which indicates its important role in fibrosis-related tumorigenesis ${ }^{[93,94]}$. In obese children, an increased abundance of Lactobacillus strains was associated with NAFLD and NASH. while in colon cancer increased Lactobacillus abundance was related to an anti-tumor effect ${ }^{[95]}$.

\section{FUTURE PERSPECTIVES}

In its current state, HCC biomarker research is far from fulfilling its promises. Therefore, it has to be subject of future investigations to elucidate the role of technologies that might complement current biomarker-based surveillance strategies. The identification of a subset of patients at the highest risk is critical to concentrate the effort and resources of regular HCC screening ${ }^{[96]}$. Chromosomal aberrations, epigenetic abnormality, and changes of gene expression are involved in hepatocarcinogenesis. Besides microbiome analysis, omics profiling (e.g., transcriptomics, proteomics, metabolomics) has been derived using several candidate HCC risk biomarkers which could refine HCC screening by enabling individual risk-stratified patient management. High-throughput omics technologies have been widely applied, aiming at the discovery of candidate biomarkers. Different types of biomolecules have been explored as sources of information to predict HCC risk. Transcriptomic dysregulations in chronic hepatopathies capture the functional molecular status supporting carcinogenesis. Circulating nucleic acids, proteins, and metabolites could serve as measures of molecular HCC risk. Large amounts of data on genetic and epigenetic abnormalities, gene expression profiles, and proteomics are available. Here, bioinformatics and network medicine increasingly play a pivotal role to organize and analyze the accumulated data ${ }^{[97]}$. Those analyses may facilitate the identification of a distinct niche of application for each individual biomarker.

\section{CONCLUSION}

HCC surveillance, in line with guidelines, significantly improves survival outcomes due to a higher proportion of patients diagnosed in an earlier stage of HCC, allowing for curative treatment options. According to most international guideline, recommendations have considered ultrasound as the method of choice for screening. US alone lacks sensitivity in the detection of small lesions, particularly in advanced cirrhosis and obesity ${ }^{[98]}$, factors that are compounded by varying skill levels of investigators and available technology. Nevertheless, numerous guidelines omitted the additional determination of AFP, despite the fact that previous trials clearly demonstrated that the utilization of AFP or biomarker-based scores such as GALAD complimentary to ultrasound resulted in a significant improvement in sensitivity while preserving high level of specificity. During the past decade, several biomarkers such as AFP-L3, DCP, osteopontin, glypican-3, and others were evaluated, however, only few markers reached longitudinal retrospective phase III development. Widely lacking are large multinational phase IV prospective screening trials confirming the benefit of the markers or their combinations for surveillance.

Facing increasing global HCC mortality, NASH has become a major risk factor for HCC development, even in the absence of cirrhosis ${ }^{[5,99,100]}$, therefore the definition of the population at risk has to be redefined for future HCC diagnostic and treatment guidelines to address this epidemiological shift. Nevertheless, costeffectiveness analyses do not support surveillance in the entire population with NAFLD who do not have cirrhosis or advanced fibrosis. Here, continued efforts of risk stratification with factors such as gender and age in conjunction with NAFLD-associated HCC risks such as PNPLA3 or TM6SF2 gene polymorphisms or distinct alterations of the microbiome and bile acid metabolism may facilitate a more concise definition of populations at HCC risk within this cohort.

Furthermore, diabetes mellitus needs to be recognized as an individual predisposing risk factor for HCC development in the presence and even in the absence of concomitant NASH. 
In the near future, the combination of standard of care ultrasound and biomarker-based screening approaches seem to be the next step to increase sensitivity and specificity of HCC surveillance.

Among the efforts of optimizing screening algorithms, the education of gate keepers such as primary care physicians, health care associates, and also the population at risk per se is another pivotal factor to increase the rate of HCC early detection.

\section{DECLARATIONS}

\section{Authors' contributions}

Conception or design of the work: Best J, Bechmann LP

Drafting the article: Best J, Sydor S

Final approval of the version to be published: Best J, Sydor S, Bechmann LP, Canbay A

Critical revision of the article: Bechmann LP, Canbay A

\section{Availability of data and materials}

Not applicable.

\section{Financial support and sponsorship}

None.

\section{Conflicts of interest}

All authors declared that there are no conflicts of interest.

\section{Ethical approval and consent to participate}

Not applicable.

\section{Consent for publication}

Not applicable.

\section{Copyright}

(c) The Author(s) 2020.

\section{REFERENCES}

1. El-Serag HB. Hepatocellular carcinoma. N Engl J Med 2011;365:1118-27.

2. El-Serag HB, Kanwal F. Epidemiology of hepatocellular carcinoma in the United States: where are we? Where do we go? Hepatology 2014;60:1767-75.

3. Rich NE, Hester C, Odewole M, Murphy CC, Parikh ND, et al. Racial and ethnic differences in presentation and outcomes of hepatocellular carcinoma. Clin Gastroenterol Hepatol 2019;17:551-9.e1.

4. De Minicis S, Agostinelli L, Rychlicki C, Sorice GP, Saccomanno S, et al. HCC development is associated to peripheral insulin resistance in a mouse model of NASH. PLoS One 2014;9:e97136.

5. White DL, Kanwal F, El-Serag HB. Association between nonalcoholic fatty liver disease and risk for hepatocellular cancer, based on systematic review. Clin Gastroenterol Hepatol 2012;10:1342-59.e2.

6. Ertle J, Dechene A, Sowa JP, Penndorf V, Herzer K, et al. Non-alcoholic fatty liver disease progresses to hepatocellular carcinoma in the absence of apparent cirrhosis. Int J Cancer 2011;128:2436-43.

7. Kudo M. Early detection and curative treatment of early-stage hepatocellular carcinoma. Clin Gastroenterol Hepatol 2005;3:S144-8.

8. Weinmann A, Koch S, Niederle IM, Schulze-Bergkamen H, Konig J, et al. Trends in epidemiology, treatment, and survival of hepatocellular carcinoma patients between 1998 and 2009: an analysis of 1066 cases of a German HCC Registry. J Clin Gastroenterol 2014;48:279-89.

9. Shah SA, Smith JK, Li Y, Ng SC, Carroll JE, et al. Underutilization of therapy for hepatocellular carcinoma in the medicare population. Cancer 2011;117:1019-26.

10. Sonnenday CJ, Dimick JB, Schulick RD, Choti MA. Racial and geographic disparities in the utilization of surgical therapy for hepatocellular carcinoma. J Gastrointest Surg 2007;11:1636-46; discussion 46. 
11. Wang Y, Luo Q, Li Y, Deng S, Wei S, et al. Radiofrequency ablation versus hepatic resection for small hepatocellular carcinomas: a metaanalysis of randomized and nonrandomized controlled trials. PLoS One 2014;9:e84484.

12. Zheng SS, Xu X, Wu J, Chen J, Wang WL, et al. Liver transplantation for hepatocellular carcinoma: Hangzhou experiences. Transplantation 2008;85:1726-32.

13. Younossi ZM, Koenig AB, Abdelatif D, Fazel Y, Henry L, et al. Global epidemiology of nonalcoholic fatty liver disease-Meta-analytic assessment of prevalence, incidence, and outcomes. Hepatology 2016;64:73-84.

14. Altekruse SF, McGlynn KA, Reichman ME. Hepatocellular carcinoma incidence, mortality, and survival trends in the United States from 1975 to 2005. J Clin Oncol 2009;27:1485-91.

15. Arends J, Bartenstein P, Bechstein W, Bernatik T, Bitzer M, et al. Leitlinienprogramm Onkologie (Deutsche Krebsgesellschaft, Deutsche Krebshilfe,AWMF): Diagnostik und Therapie des hepatozellulären Karzinoms, Kurzversion 1.0, AWMF Registrierungsnummer: 032053OL,2013. Available from: https://www.leitlinienprogramm-onkologie.de/fileadmin/user_upload/Downloads/Leitlinien/HCC/S3-HCCOL-Langversion-V1.0.pdf. [Last accessed on 14 Aug 2020]

16. Heimbach JK, Kulik LM, Finn RS, Sirlin CB, Abecassis MM, et al. AASLD guidelines for the treatment of hepatocellular carcinoma. Hepatology 2018;67:358-80.

17. EASL-EORTC clinical practice guidelines: management of hepatocellular carcinoma. J Hepatol 2018;56:908-43.

18. Omata M, Cheng AL, Kokudo N, Kudo M, Lee JM, et al. Asia-Pacific clinical practice guidelines on the management of hepatocellular carcinoma: a 2017 update. Hepatol Int 2017;11:317-70.

19. Omata M, Lesmana LA, Tateishi R, Chen PJ, Lin SM, et al. Asian pacific association for the study of the liver consensus recommendations on hepatocellular carcinoma. Hepatol Int 2010;4:439-74.

20. Kudo M, Izumi N, Kokudo N, Matsui O, Sakamoto M, et al. Management of hepatocellular carcinoma in Japan: Consensus-Based Clinical Practice Guidelines proposed by the Japan Society of Hepatology (JSH) 2010 updated version. Dig Dis 2011;29:339-64.

21. Bruix J, Sherman M, Practice Guidelines Committee AAftSoLD. Management of hepatocellular carcinoma. Hepatology 2005;42:1208-36.

22. Bruix J, Sherman M. Management of hepatocellular carcinoma: an update. Hepatology 2011;53:1020-2.

23. Bolondi L, Sofia S, Siringo S, Gaiani S, Casali A, et al. Surveillance programme of cirrhotic patients for early diagnosis and treatment of hepatocellular carcinoma: a cost effectiveness analysis. Gut 2001;48:251-9.

24. Dyson J, Jaques B, Chattopadyhay D, Lochan R, Graham J, et al. Hepatocellular cancer: the impact of obesity, type 2 diabetes and a multidisciplinary team. J Hepatol 2014;60:110-7.

25. Siegel AB, Zhu AX. Metabolic syndrome and hepatocellular carcinoma: two growing epidemics with a potential link. Cancer 2009;115:5651-61.

26. Tzartzeva K, Obi J, Rich NE, Parikh ND, Marrero JA, et al. Surveillance imaging and alpha fetoprotein for early detection of hepatocellular carcinoma in patients with cirrhosis: a meta-analysis. Gastroenterology 2018;154:1706-18.e1.

27. Araujo AR, Rosso N, Bedogni G, Tiribelli C, Bellentani S. Global epidemiology of non-alcoholic fatty liver disease/non-alcoholic steatohepatitis: what we need in the future. Liver Int 2018;38 Suppl 1:47-51.

28. Angulo P. Nonalcoholic fatty liver disease. N Engl J Med 2002;346:1221-31.

29. Vernon G, Baranova A, Younossi ZM. Systematic review: the epidemiology and natural history of non-alcoholic fatty liver disease and non-alcoholic steatohepatitis in adults. Aliment Pharmacol Ther 2011;34:274-85.

30. Wong RJ, Cheung R, Ahmed A. Nonalcoholic steatohepatitis is the most rapidly growing indication for liver transplantation in patients with hepatocellular carcinoma in the U.S. Hepatology 2014;59:2188-95.

31. Younossi Z, Stepanova M, Ong JP, Jacobson IM, Bugianesi E, et al, Global Nonalcoholic Steatohepatitis Council. Nonalcoholic steatohepatitis is the fastest growing cause of hepatocellular carcinoma in liver transplant candidates. Clin Gastroenterol Hepatol 2019;17:748-55.e3.

32. Kanwal F, Kramer JR, Mapakshi S, Natarajan Y, Chayanupatkul M, et al. Risk of hepatocellular cancer in patients with non-alcoholic fatty liver disease. Gastroenterology 2018;155:1828-37.e2.

33. Weinmann A, Alt Y, Koch S, Nelles C, Duber C, et al. Treatment and survival of non-alcoholic steatohepatitis associated hepatocellular carcinoma. BMC Cancer 2015;15:210.

34. Di Bisceglie AM, Hoofnagle JH. Elevations in serum alpha-fetoprotein levels in patients with chronic hepatitis B. Cancer 1989;64:2117-20.

35. Di Bisceglie AM, Sterling RK, Chung RT, Everhart JE, Dienstag JL, et al. Serum alpha-fetoprotein levels in patients with advanced hepatitis C: results from the HALT-C trial. J Hepatol 2005;43:434-41.

36. Sherman M, Peltekian KM, Lee C. Screening for hepatocellular carcinoma in chronic carriers of hepatitis B virus: incidence and prevalence of hepatocellular carcinoma in a North American urban population. Hepatology 1995;22:432-8.

37. Li D, Mallory T, Satomura S. AFP-L3: a new generation of tumor marker for hepatocellular carcinoma. Clin Chim Acta 2001;313:15-9.

38. Liebman HA, Furie BC, Tong MJ, Blanchard RA, Lo KJ, et al. Des-gamma-carboxy (abnormal) prothrombin as a serum marker of primary hepatocellular carcinoma. N Engl J Med 1984;310:1427-31.

39. Shang S, Plymoth A, Ge S, Feng Z, Rosen HR, et al. Identification of osteopontin as a novel marker for early hepatocellular carcinoma. Hepatology 2012;55:483-90.

40. Wang XY, Degos F, Dubois S, Tessiore S, Allegretta M, et al. Glypican-3 expression in hepatocellular tumors: diagnostic value for preneoplastic lesions and hepatocellular carcinomas. Hum Pathol 2006;37:1435-41.

41. Abelev GI, Perova SD, Khramkova NI, Postnikova ZA, Irlin IS. Production of embryonal alpha-globulin by transplantable mouse hepatomas. Transplantation 1963;1:174-80.

42. Daniele B, Bencivenga A, Megna AS, Tinessa V. Alpha-fetoprotein and ultrasonography screening for hepatocellular carcinoma. 
Gastroenterology 2004;127:S108-12.

43. Kumada T, Nakano S, Takeda I, Kiriyama S, Sone Y, et al. Clinical utility of Lens culinaris agglutinin-reactive alpha-fetoprotein in small hepatocellular carcinoma: special reference to imaging diagnosis. J Hepatol 1999;30:125-30.

44. Yamashita F, Tanaka M, Satomura S, Tanikawa K. Prognostic significance of Lens culinaris agglutinin A-reactive alpha-fetoprotein in small hepatocellular carcinomas. Gastroenterology 1996;111:996-1001.

45. Khien VV, Mao HV, Chinh TT, Ha PT, Bang MH, et al. Clinical evaluation of lentil lectin-reactive alpha-fetoprotein-L3 in histologyproven hepatocellular carcinoma. Int J Biol Markers 2001;16:105-11.

46. Taketa K, Okada S, Win N, Hlaing NK, Wind KM. Evaluation of tumor markers for the detection of hepatocellular carcinoma in Yangon General Hospital, Myanmar. Acta Med Okayama 2002;56:317-20.

47. Marrero JA, Su GL, Wei W, Emick D, Conjeevaram HS, et al. Des-gamma carboxyprothrombin can differentiate hepatocellular carcinoma from nonmalignant chronic liver disease in american patients. Hepatology 2003;37:1114-21.

48. Volk ML, Hernandez JC, Su GL, Lok AS, Marrero JA. Risk factors for hepatocellular carcinoma may impair the performance of biomarkers: a comparison of AFP, DCP, and AFP-L3. Cancer Biomark 2007;3:79-87.

49. Yuen MF, Lai CL. Serological markers of liver cancer. Best Pract Res Clin Gastroenterol 2005;19:91-9.

50. Toyoda H, Kumada T, Tada T, Kaneoka Y, Maeda A, et al. Clinical utility of highly sensitive Lens culinaris agglutinin-reactive alphafetoprotein in hepatocellular carcinoma patients with alpha-fetoprotein $<20 \mathrm{ng} / \mathrm{mL}$. Cancer Sci 2011;102:1025-31.

51. Choi JY, Jung SW, Kim HY, Kim M, Kim Y, et al. Diagnostic value of AFP-L3 and PIVKA-II in hepatocellular carcinoma according to total-AFP. World J Gastroenterol 2013;19:339-46.

52. Koike Y, Shiratori Y, Sato S, Obi S, Teratani T, et al. Des-gamma-carboxy prothrombin as a useful predisposing factor for the development of portal venous invasion in patients with hepatocellular carcinoma: a prospective analysis of 227 patients. Cancer 2001;91:561-9.

53. Lok AS, Seeff LB, Morgan TR, di Bisceglie AM, Sterling RK, et al. Incidence of hepatocellular carcinoma and associated risk factors in hepatitis C-related advanced liver disease. Gastroenterology 2009;136:138-48.

54. Johnson PJ, Pirrie SJ, Cox TF, Berhane S, Teng M, et al. The detection of hepatocellular carcinoma using a prospectively developed and validated model based on serological biomarkers. Cancer Epidemiol Biomarkers Prev 2014;23:144-53.

55. Chan SL, Mo F, Johnson PJ, Siu DY, Chan MH, et al. Performance of serum alpha-fetoprotein levels in the diagnosis of hepatocellular carcinoma in patients with a hepatic mass. HPB (Oxford) 2014;16:366-72.

56. Berhane S, Toyoda H, Tada T, Kumada T, Kagebayashi C, et al. Role of the GALAD and BALAD-2 serologic models in diagnosis of hepatocellular carcinoma and prediction of survival in patients. Clin Gastroenterol Hepatol 2016;14:875-86.e6.

57. Best J, Bilgi H, Heider D, Schotten C, Manka P, et al. The GALAD scoring algorithm based on AFP, AFP-L3, and DCP significantly improves detection of BCLC early stage hepatocellular carcinoma. Z Gastroenterol 2016;54:1296-305.

58. Best J, Bechmann LP, Sowa JP, Sydor S, Dechene A, et al. GALAD score detects early hepatocellular carcinoma in an international cohort of patients with nonalcoholic steatohepatitis. Clin Gastroenterol Hepatol 2020;18:728-35.e4.

59. Fedarko NS, Jain A, Karadag A, Van Eman MR, Fisher LW. Elevated serum bone sialoprotein and osteopontin in colon, breast, prostate, and lung cancer. Clin Cancer Res 2001;7:4060-6.

60. Zhao J, Dong L, Lu B, Wu G, Xu D, et al. Down-regulation of osteopontin suppresses growth and metastasis of hepatocellular carcinoma via induction of apoptosis. Gastroenterology 2008;135:956-68.

61. Kim J, Ki SS, Lee SD, Han CJ, Kim YC, et al. Elevated plasma osteopontin levels in patients with hepatocellular carcinoma. Am J Gastroenterol 2006;101:2051-9.

62. Wan HG, Xu H, Gu YM, Wang H, Xu W, et al. Comparison osteopontin vs AFP for the diagnosis of HCC: a meta-analysis. Clin Res Hepatol Gastroenterol 2014;38:706-14.

63. Li J, Chen X, Dai M, Huang S, Chen J, et al. Diagnostic accuracy of osteopontin plus alpha-fetoprotein in the hepatocellular carcinoma: a meta-analysis. Clin Res Hepatol Gastroenterol 2017;41:543-53.

64. Filmus J, Capurro M. The role of glypican-3 in the regulation of body size and cancer. Cell Cycle 2008;7:2787-90.

65. Capurro M, Wanless IR, Sherman M, Deboer G, Shi W, et al. Glypican-3: a novel serum and histochemical marker for hepatocellular carcinoma. Gastroenterology 2003;125:89-97.

66. Capurro MI, Xiang YY, Lobe C, Filmus J. Glypican-3 promotes the growth of hepatocellular carcinoma by stimulating canonical Wnt signaling. Cancer Res 2005;65:6245-54.

67. Nakatsura T, Yoshitake Y, Senju S, Monji M, Komori H, et al. Glypican-3, overexpressed specifically in human hepatocellular carcinoma, is a novel tumor marker. Biochem Biophys Res Commun 2003;306:16-25.

68. Jia X, Liu J, Gao Y, Huang Y, Du Z. Diagnosis accuracy of serum glypican-3 in patients with hepatocellular carcinoma: a systematic review with meta-analysis. Arch Med Res 2014;45:580-8.

69. Qiao SS, Cui ZQ, Gong L, Han H, Chen PC, et al. Simultaneous measurements of serum AFP, GPC-3 and HCCR for diagnosing hepatocellular carcinoma. Hepatogastroenterology 2011;58:1718-24.

70. Yang JD, Addissie BD, Mara KC, Harmsen WS, Dai J, et al. GALAD score for hepatocellular carcinoma detection in comparison to liver ultrasound and proposal of GALADUS score. Cancer Epidemiol Biomarkers Prev 2019;28:531-8.

71. Hassan MM, Kaseb A, Etzel CJ, El-Serag H, Spitz MR, et al. Genetic variation in the PNPLA3 gene and hepatocellular carcinoma in USA: risk and prognosis prediction. Mol Carcinog 2013;52 Suppl 1:E139-47.

72. Tang S, Zhang J, Mei TT, Guo HQ, Wei XH, et al. Association of TM6SF2 rs58542926 T/C gene polymorphism with hepatocellular carcinoma: a meta-analysis. BMC Cancer 2019;19:1128.

73. Dongiovanni P, Meroni M, Longo M, Fargion S, Fracanzani AL. miRNA signature in NAFLD: a turning point for a non-invasive 
diagnosis. Int J Mol Sci 2018;19.

74. Zhang C, Wang P, Li Y, Huang C, Ni W, et al. Role of MicroRNAs in the development of hepatocellular carcinoma in nonalcoholic fatty liver disease. Anat Rec (Hoboken) 2019;302:193-200.

75. Kutlu O, Kaleli HN, Ozer E. Molecular pathogenesis of nonalcoholic steatohepatitis- (NASH-) related hepatocellular carcinoma. Can J Gastroenterol Hepatol 2018;2018:8543763.

76. Chassaing B, Etienne-Mesmin L, Gewirtz AT. Microbiota-liver axis in hepatic disease. Hepatology 2014;59:328-39.

77. Konturek PC, Harsch IA, Konturek K, Schink M, Konturek T, et al. Gut(-)Liver Axis: how do gut bacteria influence the liver? Med Sci (Basel) 2018;6.

78. Kostic AD, Xavier RJ, Gevers D. The microbiome in inflammatory bowel disease: current status and the future ahead. Gastroenterology 2014;146:1489-99.

79. Bashiardes S, Shapiro H, Rozin S, Shibolet O, Elinav E. Non-alcoholic fatty liver and the gut microbiota. Mol Metab 2016;5:782-94.

80. Turnbaugh PJ, Ley RE, Mahowald MA, Magrini V, Mardis ER, et al. An obesity-associated gut microbiome with increased capacity for energy harvest. Nature 2006;444:1027-31.

81. Khoruts A, Sadowsky MJ. Understanding the mechanisms of faecal microbiota transplantation. Nat Rev Gastroenterol Hepatol 2016;13:508-16

82. Zhou D, Pan Q, Shen F, Cao HX, Ding WJ, et al. Total fecal microbiota transplantation alleviates high-fat diet-induced steatohepatitis in mice via beneficial regulation of gut microbiota. Sci Rep 2017;7:1529.

83. de Groot PF, Frissen MN, de Clercq NC, Nieuwdorp M. Fecal microbiota transplantation in metabolic syndrome: History, present and future. Gut Microbes 2017;8:253-67.

84. Dapito DH, Mencin A, Gwak GY, Pradere JP, Jang MK, et al. Promotion of hepatocellular carcinoma by the intestinal microbiota and TLR4. Cancer Cell 2012;21:504-16.

85. Yoshimoto S, Loo TM, Atarashi K, Kanda H, Sato S, et al. Obesity-induced gut microbial metabolite promotes liver cancer through senescence secretome. Nature 2013;499:97-101.

86. Ponziani FR, Bhoori S, Castelli C, Putignani L, Rivoltini L, et al. Hepatocellular carcinoma is associated with gut microbiota profile and inflammation in nonalcoholic fatty liver disease. Hepatology 2019;69:107-20.

87. Schonewille M, de Boer JF, Groen AK. Bile salts in control of lipid metabolism. Curr Opin Lipidol 2016;27:295-301.

88. Mouzaki M, Wang AY, Bandsma R, Comelli EM, Arendt BM, et al. Bile acids and dysbiosis in non-alcoholic fatty liver disease. PLoS One 2016;11:e0151829.

89. Neuschwander-Tetri BA, Van Natta ML, Tonascia J, Brunt EM, Kleiner DE. Trials of obeticholic acid for non-alcoholic steatohepatitis Authors' reply. Lancet 2015;386:28-9.

90. Liu Q, Li F, Zhuang Y, Xu J, Wang J, et al. Alteration in gut microbiota associated with hepatitis B and non-hepatitis virus related hepatocellular carcinoma. Gut Pathog 2019;11:1.

91. Sydor S, Best J, Messerschmidt I, Manka P, Vilchez-Vargas R. Altered microbiota diversity and bile acid signaling in cirrhotic and noncirrhotic NASH-HCC. Clin Transl Gastroenterol 2020;11:e00131.

92. Jiao N, Baker SS, Chapa-Rodriguez A, Liu W, Nugent CA, et al. Suppressed hepatic bile acid signalling despite elevated production of primary and secondary bile acids in NAFLD. Gut 2018;67:1881-91.

93. Bechmann LP, Kocabayoglu P, Sowa JP, Sydor S, Best J, et al. Free fatty acids repress small heterodimer partner (SHP) activation and adiponectin counteracts bile acid-induced liver injury in superobese patients with nonalcoholic steatohepatitis. Hepatology 2013;57:1394406.

94. Caussy C, Tripathi A, Humphrey G, Bassirian S, Singh S, et al. A gut microbiome signature for cirrhosis due to nonalcoholic fatty liver disease. Nat Commun 2019;10:1406.

95. Nowak A, Paliwoda A, Blasiak J. Anti-proliferative, pro-apoptotic and anti-oxidative activity of Lactobacillus and Bifidobacterium strains: a review of mechanisms and therapeutic perspectives. Crit Rev Food Sci Nutr 2019;59:3456-67.

96. Fujiwara N, Qian T, Koneru B, Hoshida Y. Omics-derived hepatocellular carcinoma risk biomarkers for precision care of chronic liver diseases. Hepatol Res 2020;50:817-30.

97. Pei Y, Zhang T, Renault V, Zhang X. An overview of hepatocellular carcinoma study by omics-based methods. Acta Biochim Biophys Sin (Shanghai) 2009;41:1-15.

98. Del Poggio P, Olmi S, Ciccarese F, Di Marco M, Rapaccini GL, et al. Factors that affect efficacy of ultrasound surveillance for early stage hepatocellular carcinoma in patients with cirrhosis. Clin Gastroenterol Hepatol 2014;12:1927-33.e2.

99. Chagas AL, Kikuchi LO, Oliveira CP, Vezozzo DC, Mello ES, et al. Does hepatocellular carcinoma in non-alcoholic steatohepatitis exist in cirrhotic and non-cirrhotic patients? Braz J Med Biol Res 2009;42:958-62.

100. Kawada N, Imanaka K, Kawaguchi T, Tamai C, Ishihara R, et al. Hepatocellular carcinoma arising from non-cirrhotic nonalcoholic steatohepatitis. J Gastroenterol 2009;44:1190-4. 